\title{
Segmentation and Grade Prediction of Colon Cancer Digital Pathology Images Across Multiple Institutions
}

\author{
Saima Rathore ${ }^{1,2, *}$, Muhammad Aksam Iftikhar ${ }^{3}$, Ahmad Chaddad $^{4}{ }^{(}$, Tamim Niazi ${ }^{4}$, \\ Thomas Karasic ${ }^{5}$ and Michel Bilello ${ }^{1,2}$ \\ 1 Center for Biomedical Image Computing and Analytics, University of Pennsylvania, \\ Philadelphia, PA 19104, USA; michel.bilello@uphs.upenn.edu \\ 2 Department of Radiology, Perelman School of Medicine, University of Pennsylvania, \\ Philadelphia, PA 19104, USA \\ 3 Department of Computer Science, COMSATS University Islamabad, Lahore Campus, \\ Lahore 54000, Pakistan; aksam.iftikhar@gmail.com \\ 4 Division of Radiation Oncology, Department of Oncology, McGill University, Montreal, \\ QC H3S 1Y9, Canada; ahmad.chaddad@mail.mcgill.ca (A.C.); tniazi@jgh.mcgill.ca (T.N.) \\ 5 Department of Medicine, Division of Hematology/Oncology, University of Pennsylvania, \\ Philadelphia, PA 19104, USA; Thomas.Karasic@pennmedicine.upenn.edu \\ * Correspondence: saima.rathore@pennmedicine.upenn.edu; Tel.: +1-240-753-9111
}

Received: 6 September 2019; Accepted: 17 October 2019; Published: 1 November 2019

check for updates

\begin{abstract}
Distinguishing benign from malignant disease is a primary challenge for colon histopathologists. Current clinical methods rely on qualitative visual analysis of features such as glandular architecture and size that exist on a continuum from benign to malignant. Consequently, discordance between histopathologists is common. To provide more reliable analysis of colon specimens, we propose an end-to-end computational pathology pipeline that encompasses gland segmentation, cancer detection, and then further breaking down the malignant samples into different cancer grades. We propose a multi-step gland segmentation method, which models tissue components as ellipsoids. For cancer detection/grading, we encode cellular morphology, spatial architectural patterns of glands, and texture by extracting multi-scale features: (i) Gland-based: extracted from individual glands, (ii) local-patch-based: computed from randomly-selected image patches, and (iii) image-based: extracted from images, and employ a hierarchical ensemble-classification method. Using two datasets (Rawalpindi Medical College (RMC), $n=174$ and gland segmentation (GlaS), $n=165$ ) with three cancer grades, our method reliably delineated gland regions (RMC $=87.5 \%$, GlaS $=88.4 \%)$, detected the presence of malignancy $(\mathrm{RMC}=97.6 \%$, GlaS $=98.3 \%)$, and predicted tumor grade $(\mathrm{RMC}=98.6 \%$, GlaS $=98.6 \%)$. Training the model using one dataset and testing it on the other showed strong concordance in cancer detection (Train RMC - Test GlaS $=94.5 \%$, Train GlaS - Test RMC $=93.7 \%$ ) and grading (Train RMC - Test GlaS $=95 \%$, Train GlaS - Test RMC $=95 \%$ ) suggesting that the model will be applicable across institutions. With further prospective validation, the techniques demonstrated here may provide a reproducible and easily accessible method to standardize analysis of colon cancer specimens.
\end{abstract}

Keywords: hierarchical classification; gland segmentation; colon cancer detection; colon cancer grading

\section{Introduction}

Distinguishing invasive cancer from premalignant dysplasia or benign proliferation is a fundamental task of pathologists examining colon specimens, and proper diagnosis and grading is essential to guide treatment. One of the primary challenges in colon histopathology is interand intra-observer variability that can significantly alter treatment decisions [1,2]. Review by expert 
pathologists with subspecialty training in gastrointestinal malignancy is the gold standard for diagnosis, but such second opinions are labor intensive, slow, and frequently unavailable in many resource-poor areas. With digitized images of histology slides becoming increasingly ubiquitous, automated digital pathology offers an expedited and viable solution to this problem [3-5].

Digital analysis of histology images enables extraction of quantitative morphological features, which can be used for computer-assisted grading of cancer, making the grading process more objective and reproducible [6,7]. For colorectal cancers, computer-aided diagnostic systems must analyze several morphological features of intestinal glands, including cellular architecture, gland formation, and stromal components (Figure 1). Normal colon biopsy samples have a typical and uniform glandular arrangement, but malignant tumor samples have a wide and heterogenous spectrum of disruption of standard histologic features. Our research aims to exploit such variations to address three main components of automated colon cancer diagnosis: (i) Automated gland segmentation, (ii) colon cancer detection, and (iii) colon cancer grading. It is important to note that cancer detection, in this study, refers to classifying between normal and malignant samples, whereas cancer grading, refers to further breaking down the malignant samples into different cancer grades.

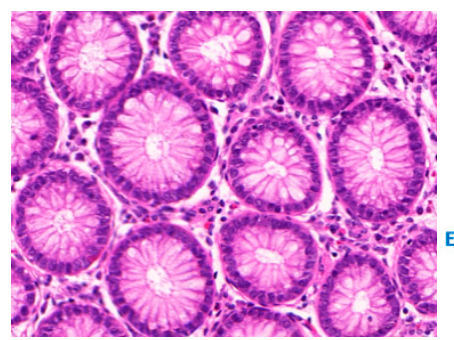

(a)

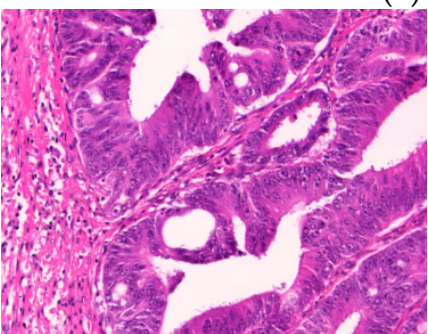

(c)

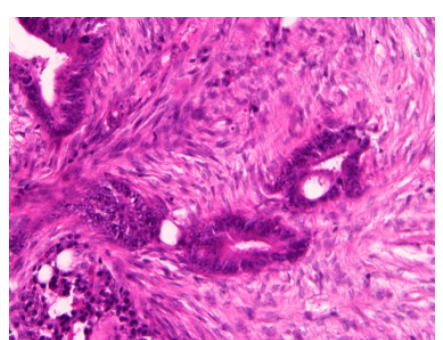

(d)

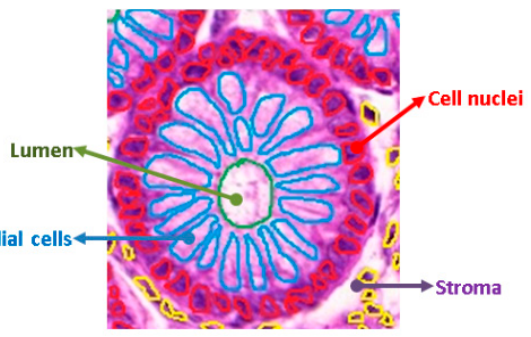

(b)

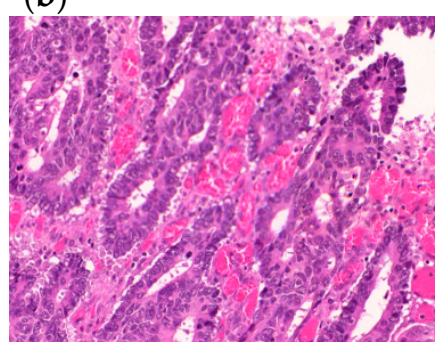

(e)

Figure 1. Example images: (a) Normal colon tissue, (b) detailed structure of a normal colon tissue; malignant colon tissue, (c) moderately differentiated, (d) moderately-to-poorly differentiated, and (e) poorly differentiated. These tissues have been stained with hematoxylin and eosin (H\&E) stain; the hematoxylin stains cell nuclei purple, and eosin stains the extracellular matrix and cytoplasm pink, with other structures taking on different shades, hues, and combinations of these colors. (a,c-e) were captured at 20× magnification, and (b) is one glandular region cropped from image (a).

Most of the published research on gland segmentation demarcates glandular boundaries, and disregards the segmentation of internal glandular components. Moreover, the existing studies on automated analysis of histologic colon cancer specimens are based on global image features, and such analyses may miss important local details that may alter the diagnosis or treatment recommendations [3,8-11]. Prior automated systems have also typically focused on separate analyses for segmentation, cancer detection, and cancer grading [12-14], and have rarely developed end-to-end pipelines.

In this paper, we propose a robust gland segmentation method for segmentation of glands and their internal structures such as epithelial cells, nuclei and lumen. We also propose a novel hierarchical classification method exploiting features at multiple image scales for colon cancer detection and grade prediction. We aim to build an end-to-end computational pathology pipeline (Figure 2) for histologic 
colon cancer detection and grade prediction by incorporating gland segmentation, cancer detection, and grading into a single automated analysis. This study contributes in five different ways:

1. A robust gland segmentation method that exploits the organizational appearance of colon glands to demarcate gland boundaries and to segment internal glandular structures, including epithelial cells, nuclei and lumen, and yields promising results for multiple datasets. The radiomic features extracted from the segmented structures were later used in the classification.

2. A multi-scale feature extraction mechanism comprising: (i) Gland-based features: extracted from individual glands, which are segmented by the proposed gland segmentation method, (ii) local-patch-based features: computed from randomly-selected patches of each histologic colon image, and (iii) image-based features: calculated by considering whole image as one unit and capturing global details.

3. Novel gland-based features, which encode morphometric properties of epithelial cells and lumen and combine them with the spatial distribution of the nuclei in relationship to stroma and lumen.

4. A multi-level hierarchical classification methodology to divide large-scale classification problem into a set of small-scale and easier-to-solve problems. In the proposed framework, the first-level classification produces probability estimates based on each feature type (image-, gland-, and local-patch-based features), and the second-level is an ensemble of various support vector machine (SVM) classifiers trained on the probability estimates generated by the first-level.

5. Demonstration of the generalizability of the proposed pipeline across multiple datasets.

1. Colon histology images

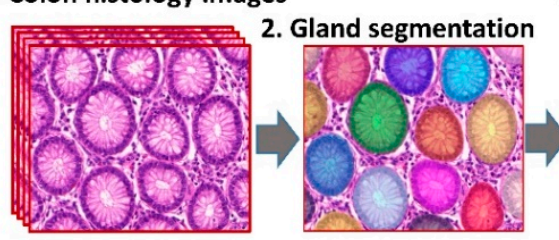

3. Quantification of tissue morphology (Feature extraction)
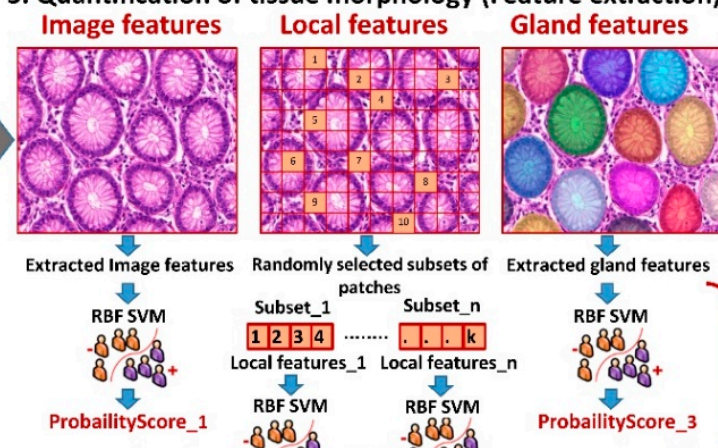

ProbailityScore_1

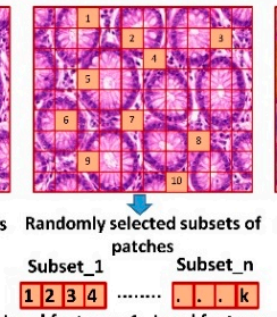

Local features_1 Local features
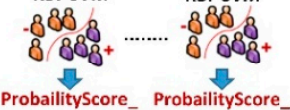

Subset_1 Subset_n

robability of classifier with

greatest confidence

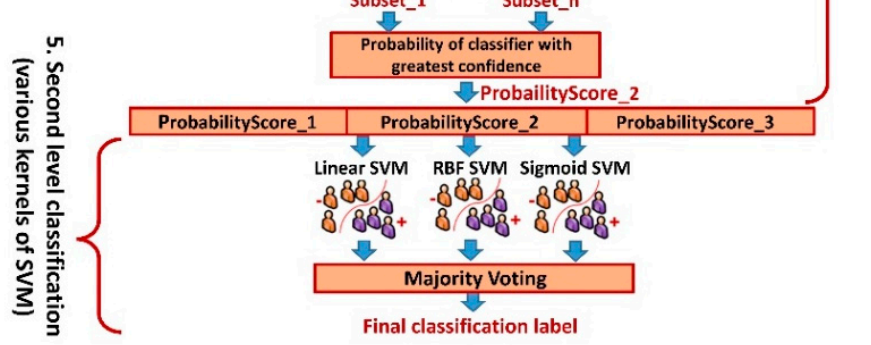

Final classification label

Figure 2. Schematic overview of the proposed methodology: (1) Colon tissue histology images, (2) gland segmentation using the proposed multi-step method, (3) quantification of tissue morphology, (4) first level of classification using radial basis function (RBF) kernel of support vector machine (SVM), and (5) second level of classification using majority voting based on the predictions of various weak classifiers such as linear, RBF, and sigmoid kernel of SVM.

\subsection{Related Work}

This section presents a review of contemporary literature in the three research directions on colon cancer diagnosis, which have also been investigated in the current work, i.e., automated gland segmentation, colon cancer detection and grading. 
Glandular structures in histopathology images can be segmented either by using generic [15] or specialized methods [16-18]. Graph-based methods, which rely on generating graphs from glandular structures, have been the most common method for gland segmentation. For example, Demir et al. decomposed the glandular structures in their primitive objects and utilized the organizational properties of these objects instead of traditional pixel-based properties [16]. The approach relies on a region growing procedure, where the gland seeds are determined based on a graph constructed from the nucleus and lumen objects. The seeds are grown using another object-graph constructed on the nucleus objects alone. The final boundary of glands is obtained based on the locations of the nucleus objects and a false-positive elimination process based on information of the segmented grown regions. Similarly, Fu et al. proposed a graph-based GlandVision algorithm [19]. Using the random field modelling in the polar space, the gland contours were extracted based on an inference strategy that approximates a circular graph using two chain graphs. Then, a support vector regressor based on visual features was used to verify that the extracted contour belonged to a true gland.

Some recent research efforts have also employed deep neural networks for gland segmentation. To this end, Kainz et al. [18] presented a deep convolutional neural network based pixel classifier for semantic segmentation of colon gland images. Two 7-layer convolutional neural networks (CNNs) were used to predict whether individual pixels belonged to normal or malignant glands. These predictions were then normalized based on weighted total variation using a figure-ground segmentation approach. Wenqi et al. [20] also employed fine-tuned CNNs for segmenting glandular structures, but combined them with an SVM classifier based on traditional radiomic features. Recently, for gland segmentation, Chen et al. proposed a deep contour-aware network that uses a unified multi-task learning framework, exploits multi-level contextual features, and employs an auxiliary supervision method to solve the problem of vanishing gradients [21]. Graham et al. proposed a CNN that counters the information loss incurred in max-pooling layers by re-introducing the original image at multiple points within the network [17]. They used atrous spatial pyramid pooling with varying dilation rates for preserving the resolution and multi-level aggregation, and introduced random transformations during test time for an enhanced segmentation result that concurrently generates an uncertainty map and highlights ambiguous areas. We aim to improve upon this prior art by proposing a gland segmentation algorithm that not only delineates gland boundaries, but also demarcates the internal glandular structures using a multi-step process based on the geometrical/morphological properties of the structures.

Several radiomic methods exist in the literature to distinguish benign and malignant colon lesions. For example, Masood et al. [22] investigated local-binary-patterns along with Gaussian SVM to produce reasonable classification results. Classification accuracy can be improved using an ensemble of different classifiers and hybrid combinations of discriminative features. For example, Rathore et al. [3] employed different ensemble classifiers such as rotation boost, rotation forest and random forest on a hybrid feature set, comprising white run-length and area features, and achieved better classification accuracy than from any individual classifier or feature. Similarly, combining a textural analysis of color components with a histogram-of-oriented-gradients improved classification performance [23]. Also, chaddad et al. proposed several radiomic pipeline to evaluate the continuum of colorectal cancer using various types of shape and texture features with multi classifier models [12-14]. Considered the CNN models, the classification accuracy was improved compared to the conventional classifier models [13].

Similar to automated gland segmentation, some researchers have employed graph-based techniques to standardize colon cancer grading. Altunbay et al. [9] employed the characteristic of circularity in shape of pink, purple, and white clusters of colon biopsy images. They applied a circle finding algorithm on these clusters and computed discriminative features on a graph generated from circular objects in these clusters. They validated this technique by detecting colon cancer from colon biopsy images and discriminating different cancer grades with high accuracy. Ozdemir et al. [10] presented a similar technique based on graph creation from the three clusters of colon biopsy images of normal subjects. These test graphs were compared with training graphs to determine whether tissues were normal or malignant based on the extent of correlation with the test graph. In contrast, 
Rathore et al. [11] used lumen circularity, convexity, concavity, and ratio of lumen area to the size of image and white cluster as features, which improved the accuracy of cancer grading. In a recent study, Awan et al. measured the shape of glands with a novel metric that they called the "best alignment metric" (BAM). A SVM classifier was then trained using shape features derived from BAM that yielded an accuracy of $91 \%$ in a three-class classification into normal, low grade cancer, and high grade cancer [24]. A comprehensive review further describes colon cancer segmentation, detection and grading techniques [7].

Despite the significant advances in the past two decades, end-to-end computational pathology pipelines have rarely been developed by the researchers. Our paper aims to bridge this gap and provides an end-to-end computational pathology pipeline for histologic colon cancer detection and grade prediction by incorporating gland segmentation, cancer detection, and grading into a single automated analysis.

\section{Results}

\subsection{Performance of The Proposed Gland Segmentation Method}

The segmentation results of the proposed algorithm are visually illustrated for various normal and malignant samples (Figure 3). These results demonstrate that the proposed algorithm successfully identifies glandular boundaries and properly segments glands across irregularly arranged malignant samples. The corresponding quantitative results are shown in Table 1 for two different datasets, gland segmentation (GlaS) challenge dataset, and Rawalpindi Medical College (RMC) dataset.

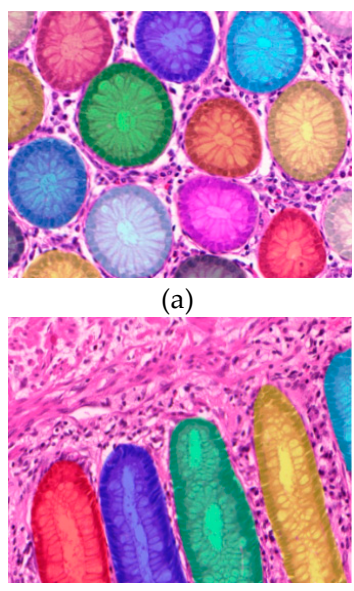

(e)

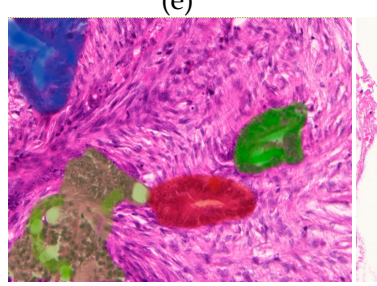

(i)

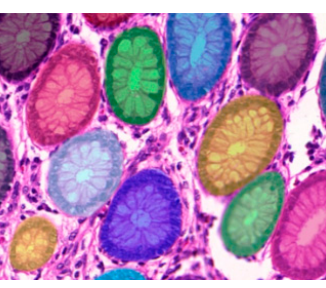

(b)

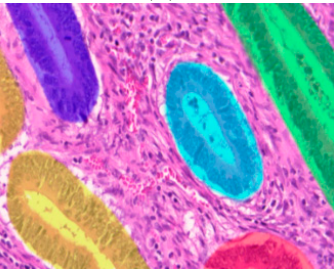

(f)

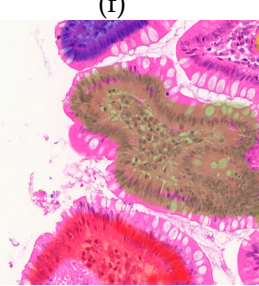

(j)

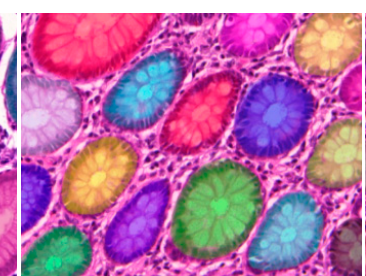

(c)

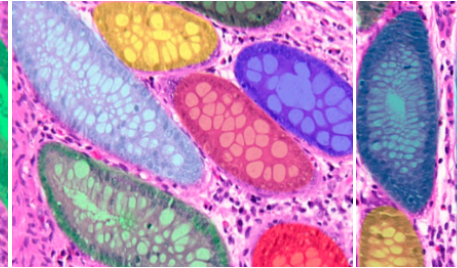

(g)

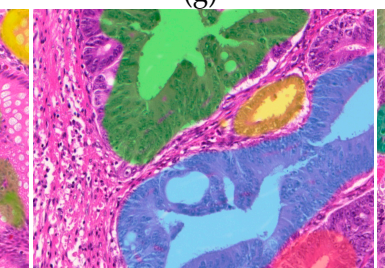

(k)

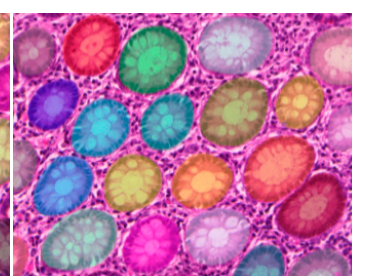

(d)

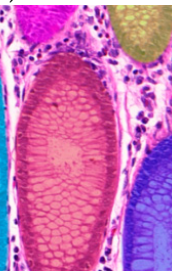

(h)

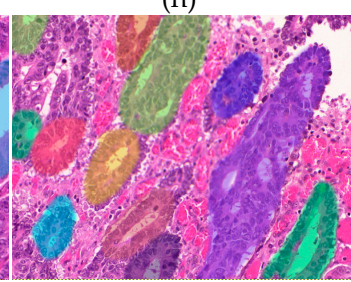

(1)

Figure 3. Example images showing the output of gland segmentation method. 1st row: normal samples, and 2nd and 3rd row: malignant samples of varying cancer grades. The method successfully captures the bounds of glandular regions and leads to good segmentation results for images in the 1st and 2 nd rows despite the fact that images have huge variations and their glands appear in less regular structures. The third row shows example cases where the method could not identify boundaries of a few glands or has under-segmented (especially in the top-left corner of $i$ and top-middle of $j$ ). All images were captured at $20 \times$ magnification. 
Table 1. Performance of the proposed gland segmentation method.

\begin{tabular}{ccc}
\hline Performance Metrics & RMC-Dataset & GlaS-Dataset \\
\hline Segmentation accuracy & 87.50 & 88.40 \\
Jaccard index & 0.86 & 0.89 \\
Dice similarity & 0.84 & 0.87 \\
Sensitivity & 0.90 & 0.92 \\
Specificity & 0.82 & 0.88 \\
F-Score & 0.88 & 0.89 \\
\hline
\end{tabular}

\subsection{Performance of The Proposed Cancer Detection and Grading Method}

Classification rates obtained using the individual features are summarized in terms of classification accuracy, sensitivity, specificity, and Matthew's correlation coefficient (MCC) for cancer detection and respective grading (Table 2). Our predictive model's accuracies in correctly classifying the normal and malignant images on the GlaS and RMC datasets were $93.7 \%$ and $92.1 \%$ for gland-based features, $93.1 \%$ and $91.5 \%$ for patch-based features, and $92.5 \%$ and $90.9 \%$ for image-based features, respectively. Similarly, the cross-validated 3-class accuracy of cancer grading on the GlaS and RMC datasets was $90.5 \%$ and $92.5 \%$ for gland-based features, $89.5 \%$ and $91.5 \%$ for patch-based features, and $89.7 \%$ and $90.7 \%$ for image-based features, respectively. No statistically significant differences of the feature extraction methods between the two datasets by McNemar Test were noted.

Table 2. Performance of individual feature extraction methods (results are shown for the ensemble classifier for the patch-based features).

\begin{tabular}{ccccccc}
\hline & \multicolumn{2}{c}{ Gland-Based } & \multicolumn{2}{c}{ Local-Patch-Based } & \multicolumn{2}{c}{ Image-Based } \\
\hline & GlaS-Dataset & RMC-Dataset & GlaS-Dataset & RMC-Dataset & GlaS-Dataset & RMC-Dataset \\
\hline \multicolumn{7}{c}{ Cancer Detection } \\
\hline Accuracy & 93.7 & 92.1 & 93.1 & 91.5 & 92.5 & 90.9 \\
Sensitivity & 92.4 & 86.8 & 91.3 & 85.2 & 92.3 & 84.5 \\
Specificity & 95.1 & 98.6 & 93.9 & 97.4 & 92.7 & 95.9 \\
MCC & 87.4 & 85.0 & 86.2 & 83.7 & 85.0 & 82.3 \\
\hline \multicolumn{7}{c}{ Cancer Grading } \\
Accuracy & 90.5 & 92.5 & 89.5 & 91.5 & 89.7 & 90.7 \\
Sensitivity & 84.4 & 89.0 & 82.9 & 87.0 & 81.5 & 85.5 \\
Specificity & 93.1 & 94.9 & 92.0 & 92.5 & 91.6 & 92.4 \\
MCC & 79.0 & 83.9 & 76.0 & 80.6 & 74.7 & 78.6 \\
\hline
\end{tabular}

The various constituents of the meta-classifier were trained based on the probability estimates generated by the first-level classification, and their output was combined using majority voting to get final predictions (Table 3). The cross-validated 2-class accuracy of cancer detection was $98.3 \%$ for the GlaS dataset and $97.6 \%$ for the RMC dataset. Similarly, the cross-validated 3-class accuracy of cancer grading was $98.6 \%$ both for GlaS and RMC datasets. All the individual classifiers performed reasonably well for both cancer detection and grading tasks, however, an optimal combination of many complementary imaging features/classifiers through a meta-classifier achieved even higher accuracy, which is statistically significant $(p<0.01)$ at $95 \%$ confidence interval. Furthermore, comparing the assessment of individual features/classifiers with the multivariate results of combined features and classifiers reveals that subtle individual features can be synthesized in an index of higher distinctive performance. 
Table 3. Performance of the meta-classifier for both data sets (results are shown for individual and ensemble classifier).

\begin{tabular}{|c|c|c|c|c|c|c|c|c|}
\hline & \multicolumn{4}{|c|}{ GlaS-Dataset } & \multicolumn{4}{|c|}{ RMC-Dataset } \\
\hline & Linear & RBF & Sigmoid & Ensemble & Linear & RBF & Sigmoid & Ensemble \\
\hline \multicolumn{9}{|c|}{ Cancer Detection } \\
\hline Accuracy & 94.3 & 95.4 & 94.8 & 98.3 & 92.1 & 93.3 & 92.7 & 97.6 \\
\hline Sensitivity & 91.3 & 92.4 & 92.4 & 97.8 & 96.7 & 98.9 & 98.9 & 98.9 \\
\hline Specificity & 97.6 & 98.8 & 97.6 & 98.8 & 86.5 & 86.5 & 85.1 & 95.9 \\
\hline MCC & 88.7 & 91.2 & 89.8 & 96.5 & 84.2 & 86.9 & 85.8 & 95.1 \\
\hline \multicolumn{9}{|c|}{ Cancer Grading } \\
\hline Accuracy & 94.2 & 94.5 & 93.7 & 98.6 & 91.3 & 93.6 & 93.5 & 98.6 \\
\hline Sensitivity & 90.7 & 91.4 & 90.4 & 97.3 & 84.7 & 87.7 & 87.8 & 97.4 \\
\hline Specificity & 95.7 & 95.9 & 95.0 & 99.0 & 93.7 & 95.7 & 95.4 & 99.0 \\
\hline MCC & 86.4 & 87.3 & 85.4 & 96.4 & 78.4 & 83.3 & 83.2 & 96.4 \\
\hline
\end{tabular}

A receiver operating characteristic (ROC) analysis was performed to verify the reliability of classification results. Area under the curve (AUC) was 0.95 and 0.99 for cancer detection of the RMC and GlaS datasets, respectively, and 0.98 and 0.96 for cancer grading of the RMC and GlaS datasets, respectively (Figure 4).
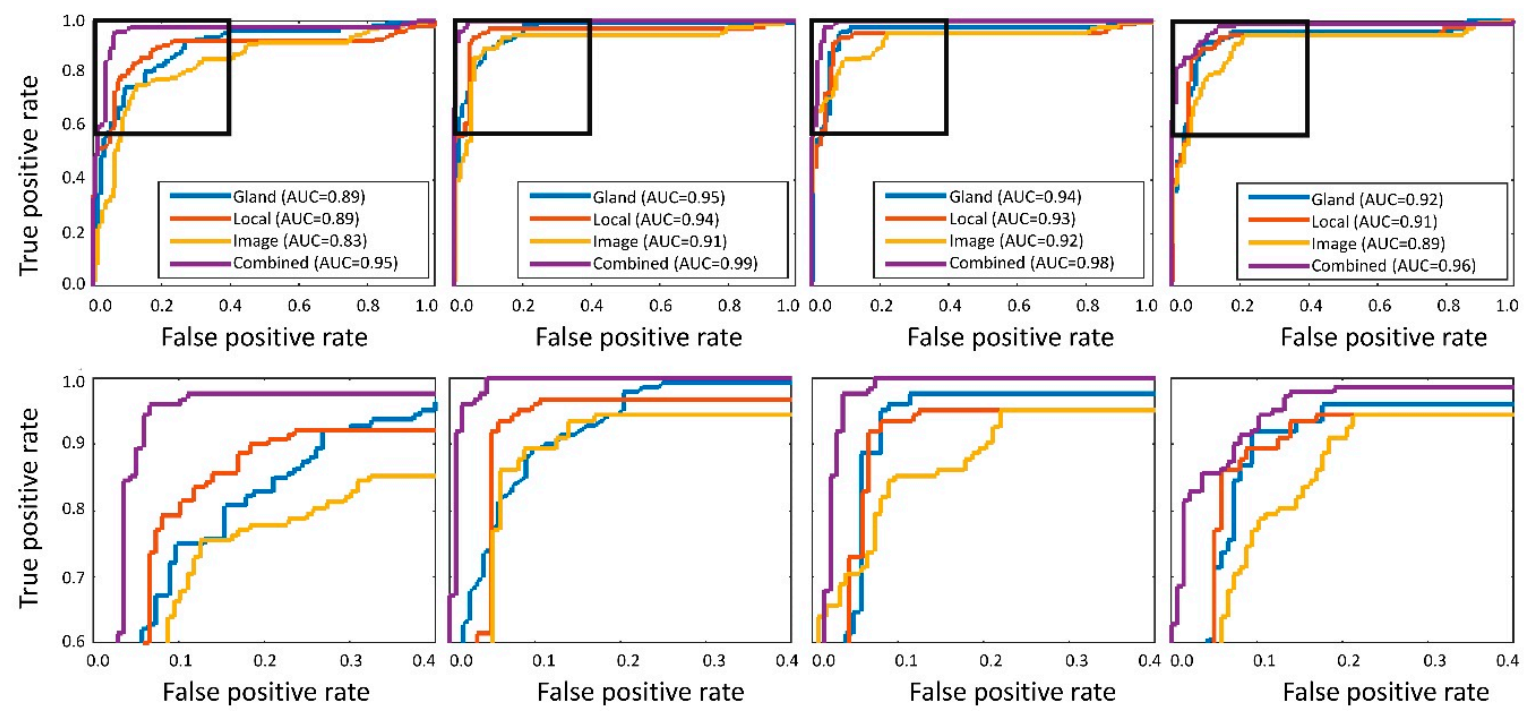

Figure 4. Top-row: Receiver operating characteristic (ROC) curve analysis and the corresponding area under the curves (AUCs) for individual feature categories and the meta-classifier. Bottom-row: zoomed in version of ROC curves. Left-to-right: Cancer detection for RMC-Dataset, cancer detection for GlaS-Dataset, cancer grading for RMC-Dataset, and cancer grading for GlaS-Dataset.

\subsection{Performance of the Proposed Cancer Detection and Grading Method across Different Institutions}

To determine the applicability of our radiomic analysis across datasets, we trained the two-stage classification framework on one dataset and tested it on the other, and vice versa (Table 4). For cancer detection, a model trained on the RMC dataset and tested on the GlaS dataset yielded an accuracy of $94.5 \%$, while a model trained on GlaS and tested on RMC yielded an accuracy of $93.7 \%$. For cancer grading, an accuracy of $95.0 \%$ was observed for both training and testing pairs. 
Table 4. Performance of the meta-classifier across multi-institutions (results are shown for individual and ensemble classifiers).

\begin{tabular}{lcccccccc}
\hline & \multicolumn{2}{c}{ Train RMC-Dataset + Test GlaS-Dataset } & \multicolumn{3}{c}{ Train GlaS-Dataset + Test RMC-Dataset } \\
\hline & Linear & RBF & Sigmoid & Ensemble & Linear & RBF & Sigmoid & Ensemble \\
\hline \multicolumn{7}{c}{ Cancer Detection } \\
\hline Accuracy & 89.7 & 91.5 & 90.9 & 94.5 & 89.7 & 90.2 & 87.9 & 93.7 \\
Sensitivity & 97.8 & 96.7 & 96.7 & 96.7 & 96.0 & 96.7 & 91.3 & 96.7 \\
Specificity & 79.7 & 85.1 & 83.8 & 91.9 & 81.7 & 82.9 & 84.1 & 90.2 \\
MCC & 79.9 & 83.1 & 81.9 & 89.0 & 79.9 & 80.9 & 75.8 & 87.4 \\
\hline \multicolumn{7}{c}{ Cancer Grading } \\
\hline Accuracy & 89.8 & 91.3 & 90.0 & 95.0 & 89.8 & 90.6 & 90.7 & 95.0 \\
Sensitivity & 82.2 & 84.5 & 81.6 & 91.6 & 84.1 & 84.7 & 86.0 & 92.8 \\
Specificity & 92.2 & 93.6 & 93.0 & 96.3 & 92.1 & 92.8 & 92.4 & 95.8 \\
MCC & 74.3 & 78.2 & 74.6 & 87.9 & 76.2 & 77.5 & 78.5 & 88.6 \\
\hline
\end{tabular}

\subsection{Interpretation of Features of Normal/Malignant Colon Tissues}

To obtain a deeper understanding of the information (features) used by SVM to provide predictions, boxplots of some predictive features were generated by dividing the patient cohort into 2 groups according to the normal/malignant status. We have selected top-most 5 features based on the weight assigned by the classifier, and compared the values of these features in normal and malignant groups. Boxplots of these features based on data from all the patients were created with the group type on $x$-axis and its value on y-axis (Figure 5). The analysis revealed that each tissue type had a unique set of radiographically interpretable features associated with it. The main findings from comparing the features of different tissue types are as follows:

1. Lower entropy values in normal colon tissue images $\left(p=5.6 \times 10^{-5}\right)$, suggestive of more uniformity and proper organizational structure of tissue compared to malignant colon tissue that shows very high entropy, indicative of more randomness and lack of any organizational structure;

2. Lower contrast values in normal colon tissue $\left(p=2.1 \times 10^{-6}\right)$, which again point towards coherent and properly organized tissue. On the other hand, the higher contrast values in malignant colon tissues show the lack of proper organizational structure;

3. Lower values of standard deviation of distances of cell nuclei from the centroid of lumen for normal colon tissue $\left(p=8.6 \times 10^{-4}\right)$ are suggestive of the fact that cell nuclei are almost equally spaced from the centroid of the lumen, whereas the same is not true for malignant colon tissue where cell nuclei are at random distances from lumen;

4. Higher values of eccentricity $\left(p=2.1 \times 10^{-3}\right)$ and compactness $\left(p=5.8 \times 10^{-4}\right)$ in normal colon tissues, consistent with the well-defined shape of normal colon tissue. On the contrary, the malignant colon tissues exhibit lower compactness and eccentricity, indicative of the migratory and deeply infiltrated tissue.
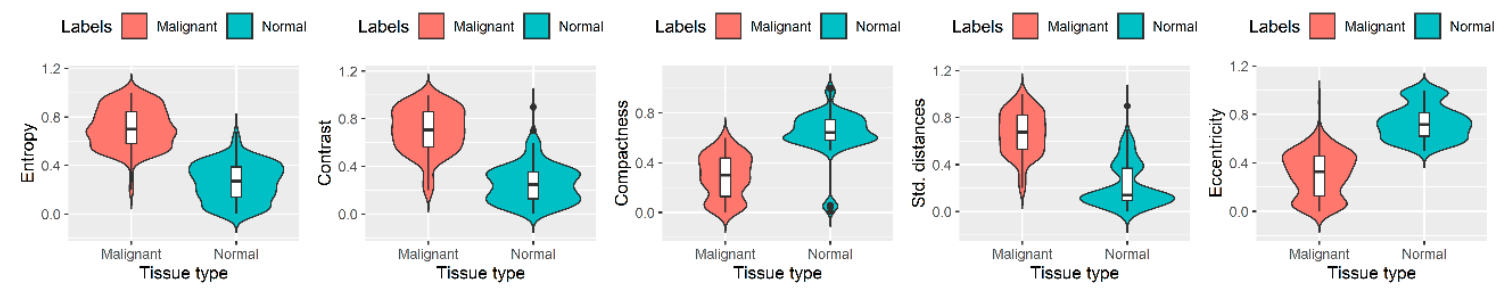

Figure 5. Interpretation of features of normal and malignant colon tissues. X-axis of the box-plots shows the tissue type, which is either normal or malignant, and the $y$-axis shows the value of the feature. The feature of standard deviation of distances (Std. distances) was scaled in the range (0-1) for better visualization. 
None of the individual features are sufficient to distinguish normal from malignant tissue but multivariate analysis through machine learning accurately classifies benign and malignant samples.

\section{Discussion}

In this paper, we have demonstrated accurate identification and grading of malignant colon cancer samples using an automated computational pathology pipeline.

The segmentation method proposed herein provided an accurate demarcation of glandular regions of the colon tissue. We want to mention that despite the prior development of several deep-learning based gland segmentation algorithms, we proposed an additional algorithm because the proposed algorithm not only demarcates boundaries but also divides glandular region into its constituent elements, that we later quantify in terms of features to use in the cancer detection and grading steps. Also, in the absence of ground-truth of internal glandular regions, training deep learning algorithms was not possible, therefore, we adopted an alternative approach comprising a series of image processing steps to segment internal glandular regions. Moreover, there are several performance advantages of the proposed algorithm over deep learning approaches. First, the processing time of this algorithm is lower than that of computationally expensive deep learning algorithm. Second, this algorithm does not need any training, and can be applied on any incoming specimen without having a pre-trained model in place. Third, the method does not need any sophisticated hardware to run, and can run on desktop machines having only CPU. The performance of the proposed algorithm on GlaS dataset (F-Score $=0.89$, Dice $=0.87$ ) for the task of outer glandular boundary segmentation is also in par with the existing methods. For example, the best performing method in MICCAI 2015 gland segmentation contest [21] reported F-Score of 0.887, and another recent study [17] reported F-Score of 0.844 and Dice of 0.836 on Test A, and F-Score of 0.914 and Dice of 0.913 on Test B datasets, respectively, released as part of MICCAI 2015 gland segmentation contest.

Our multi-feature hierarchical classification method consistently and substantially improved upon the classification performance of the individual-features-based classification methods. Similarly, the proposed ensemble frameworks, either the ensemble for patch-based features at the first-level of classification or the ensemble for meta-classifier at the second-level of classification, reinforced the classification accuracy of individual classifiers and yielded better prediction estimates. Overall, our methods achieved a high accuracy for cancer detection $(\mathrm{RMC}=97.6 \%, \mathrm{GlaS}=98.3 \%$ ) and grading ( $\mathrm{RMC}=98.6 \%$, GlaS $=98.6 \%$ ). Importantly, the features highlighted as most discriminative by the machine learning algorithm including entropy, cellular eccentricity, and nuclear displacement, are the same visual features relied upon by histopathologists to distinguish between normal and malignant tissues. Our findings are consistent with the previous literatures. For example, Chaddad et al. showed that the feature of entropy is able to discriminate between four type of pathology tissues [12]. It was also observed in another study [25] that the measure of eccentricity was a good predictor of intraepithelial neoplasia, which is a precursor state for full-blown carcinoma.

\subsection{Validation of the Proposed Method across Different Datasets}

To confirm that our methods would be applicable across multiple institutions, we trained our model on a dataset from one institution and tested it on a dataset from another institution. Previous studies, either on demarcation of malignant and benign tissue regions, or on cancer detection and grading have not undergone such validation $[11,16,19,22,26,27]$. Our findings (Tables 3 and 4) support the notion that the combination of features and machine learning classification proposed in this study allows robust classification of colon cancer datasets arising from multiple institutions, even if a new dataset comes from an institution that was not part of the training sample. The validation of our methods across datasets strongly suggests that our model will perform well in routine clinical settings where samples are much more diverse than in controlled experimental settings. 


\subsection{Importance of the Study}

The computational pathology pipeline described in this paper addresses many of the current barriers in clinical histopathology. This method offers a standardized approach to resolve intra- and inter-observer variability amongst pathologists and is easily implemented even in low resource settings with available digital scanning tools. Importantly, although this study is focused on colon cancer, the same approach could also be used for other types of cancer. For example, automated analysis may improve grading in neuroendocrine tumors, where treatment decisions are heavily reliant on accurate grading. Similarly, the speed and reproducibility of this method may improve reliability of grading in heterogeneous samples. One additional application may be to identify tumor origin by facilitating comparisons of multiple biopsy specimens from the same patient to determine the morphological concordance between a metastatic site and a primary tumor.

\subsection{Limitations and Future Work}

Our study has several limitations. First, the proposed segmentation method has specially been designed to segment the glandular regions, and is not suitable for regional separation of benign and malignant tissue. However, the method could be tailored, with appropriate modifications, to be used for regional demarcation of benign and malignant regions. Second, since our method is specially designed to decode the morphology of glands, it cannot accurately quantify the features of extremely deformed glands in very poorly-differentiated specimens. Another limitation of our study is that we used retrospective data; a prospective dataset comparing our methods to standard histopathological review would lend further validity to our model.

Beyond the scope of this work, there are several important future research directions. Previous machine learning studies have shown strong association between the CT imaging characteristics and survival of colorectal cancer patients [28] and molecular subtypes [29]. We aim to examine whether the analysis of histologic features demonstrated here can similarly predict clinical outcomes and genomic aberrations. A systematic analysis of these characteristics with the help of gland segmentation as part of automatic image analysis framework could lead to a better understanding of the relevant cancer biology as well as bring precision and accuracy into assessment and prediction of the outcome of the cancer.

\section{Materials and Methods}

\subsection{Datasets}

The pathology data were obtained from two independent sources: (i) 174 images, acquired at $10 \times$ magnification factor, were collected from pathology department of RMC, Rawalpindi, Pakistan. These images were subdivisions of 68 hematoxylin and eosin stained slides of colon resection specimens, with each slide belonging to a different patient. The hematoxylin stains cell nuclei purple, and eosin stains the extracellular matrix and cytoplasm pink, with other structures such as lumen and epithelial cells taking on shades very close to white color. The images were labeled as normal $(n=82)$ or malignant $(n=92)$ by an expert pathologist, and malignant images were further categorized into three different grades (well-differentiated $=44$, moderately-differentiated $=25$, and poorly-differentiated $=23$ ) [27]; (ii) 165 images, acquired at 20× magnification factor, were collected from the GlaS contest [30]. These images were captured from 16 hematoxylin and eosin stained slides of colon resection specimens, each from a different patient. Images were labelled by an expert pathologist as normal $(n=74)$ or malignant $(n=91)$, and malignant images were further distributed into three different grades (moderate $=47$, moderate-to-poor $=20$, poor $=24$ ) [31]. To allow for comparison between the two data sets, we converted these three categories into well-, moderately-, and poorly-differentiated grades. Contrast enhancement was applied to decrease imaging artifacts. Following contrast-enhancement, images were converted to gray-scale for further analysis. 


\subsection{The Gland Segmentation Algorithm}

A multi-step image processing method (Figure 6) was used to delineate colon glands in biopsy images:
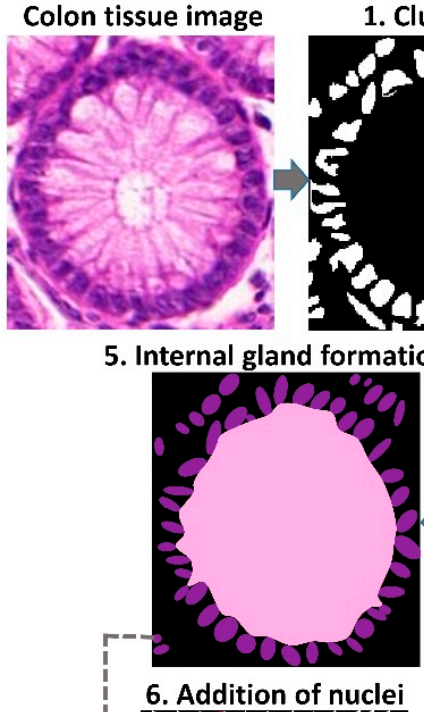

1. Clustering of tissue components

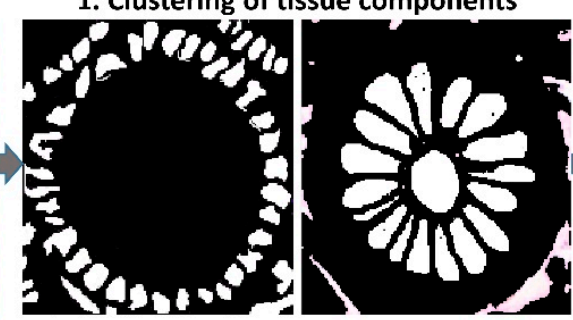

2. Ellipse fitting

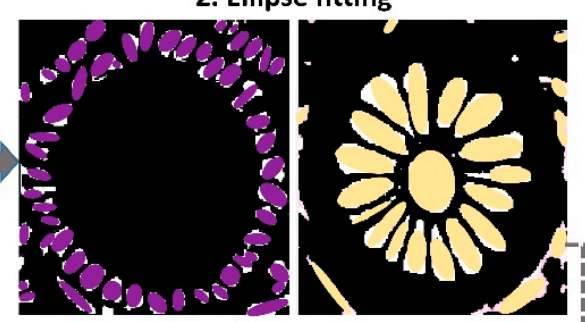

3. Identification of inner/outer tissue
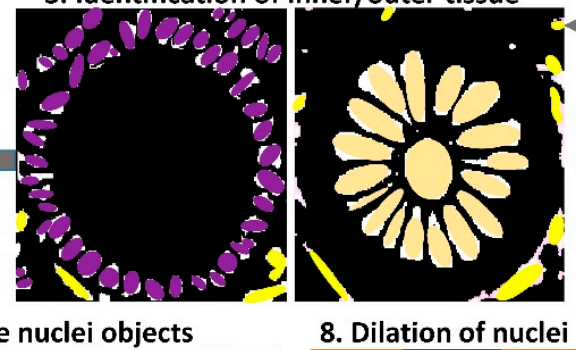

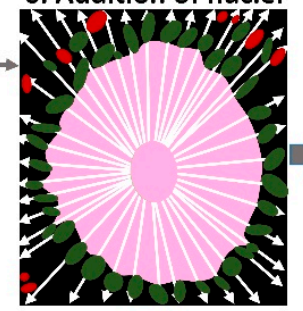

7. Removal of false nuclei objects
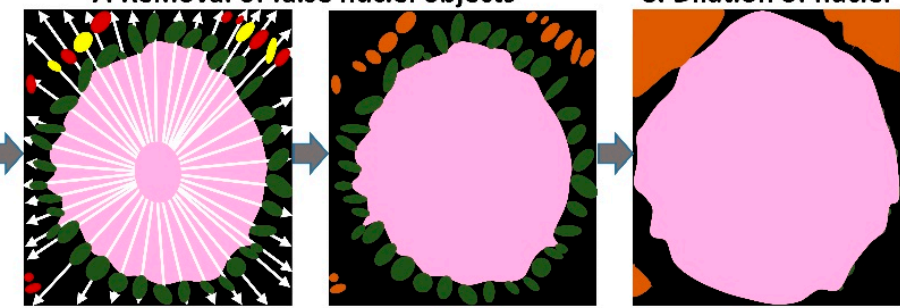

Figure 6. Schematic workflow of the proposed gland segmentation method. Figure shows one glandular region cropped from an image captured at $20 \times$ magnification.

\subsubsection{Clustering of Tissue Components}

Each image was divided into white, pink and purple clusters using the K-means algorithm. A morphological operation of erosion was performed on the clusters.

\subsubsection{Ellipse Fitting}

An ellipse-fitting algorithm [27] was used to identify purple-colored nuclei and white-colored epithelial cell cytoplasm and glandular lumina.

\subsubsection{Discrimination of True and False Glandular Components}

To improve the ellipse-fitting algorithm, hierarchical clustering was applied to discriminate between true glands and false positives. Four co-centric ellipses of different orientations were overlaid on each detected object, and the following features were analyzed: (i) Total number of detected objects; (ii) average Euclidean distance between the centers of objects; (iii) mean and standard deviation of areas and compactness of objects; and (iv) ratio of purple area to white/pink area (for purple objects), and ratio of white area to pink/purple cluster (for white objects). Hierarchical clustering, when applied separately on the features of white and purple objects, divided the purple objects into two classes: objects on the boundary of the glands (true cell nuclei) and false positive objects scattered within stroma. Similarly, the clustering of the white objects divided the objects into true epithelial cell cytoplasm and glandular lumina, and false positive white areas outside the gland. 


\subsubsection{Lumen Detection}

Following the identification of epithelial cell cytoplasm and gland lumina in the previous step, hierarchical clustering was applied to distinguish between the cytoplasm and lumen.

\subsubsection{Formation of Internal Gland Regions}

A morphological dilation of size 3 was applied on the lumen and epithelial cells in an iterative manner until no nuclei got added to the dilated region.

\subsubsection{Addition of Nuclei to the Internal Glandular Region}

A geometrical, heuristic approach was adopted in order to add nuclei to the identified glandular region containing lumina and epithelial cell cytoplasm. Radial lines were drawn from the center of lumen to each boundary point of the internal glandular region, and were extended towards the image boundary. The nuclei lying closest to the lumen on each radial line were considered candidate boundary objects (shown in green).

\subsubsection{Removal of False Nuclei}

To remove false nuclei (shown in yellow), all the candidate boundary nuclei were clustered into two classes (outliers and real boundary nuclei) by applying a hierarchical clustering method on features extracted from each identified nucleus. In particular, the difference between Euclidean distance of a certain nucleus and its 5 neighboring nuclei was used in hierarchical clustering to identify real nuclei.

\subsubsection{Dilation of Nuclei}

Once the boundary nuclei were identified, the inner gland regions were dilated until all the voxels of all the boundary nuclei were added to the inner region, thereby constituting the complete gland region.

To quantitatively measure the success of the obtained segmentation results, true + , false + , falseand true- were calculated using the manual segmentation as the gold standard and then the sensitivity, specificity, accuracy, dice similarity coefficient and Jaccard Index were computed.

\subsection{Features for Colon Cancer Detection/grading}

For each image, we extracted multiple radiomic features captured at various scales, i.e., (i) image-, (ii) gland-, and (iii) patch-based features, in order to capture various phenotypic characteristics of the tumors.

\subsubsection{Image-Based Features}

The overall (global) texture of the images was quantified using Haralick texture features to appreciate significantly different texture of normal and malignant colon tissues. These features were computed from the Spatial Gray Level Dependence (SGLD) matrix constructed from the input image as a whole. The SGLD matrix measures the frequency of co-occurrences of pairs of gray-levels at certain offset $d$ and angle $\theta$. In this work, an offset value of 1 was used at four different angles $\left(0^{\circ}, 45^{\circ}\right.$, $90^{\circ}$, and $135^{\circ}$ ) to produce different SGLD matrices of $8 \times 8$ dimensions (assuming 8 scaled gray levels in the input image). Later on, the texture features of entropy, energy, correlation, inverse difference moment, inertia, sum average, sum entropy, sum variance, difference variance, difference average, and difference entropy were computed by averaging features from all the generated matrices [32].

\subsubsection{Gland-Based Features}

The structure and shape of individual glands, which significantly vary between normal and malignant colon tissues, was also quantified to better characterize the heterogeneity by selecting the 5 largest and 5 smallest glands (in terms of area). The features extracted from each gland include: (i) 
Std. deviation of distances of epithelial cells (white) and cell nuclei (purple) from lumen (Figure $7 \mathrm{~b}, \mathrm{c}$ ). The distances are computed from centroid of each object; (ii) Standard deviation of areas of epithelial cells and cell nuclei; (iii) Ratio of the sizes of different gland components (lumen, epithelial cells, nuclei) to the total pixels of the gland, and the pair-wise ratio of all the gland components (Figure 7a); and (iv) morphological features of area, compactness, convex area, eccentricity, Euler number, major- and minor-axis length, orientation and perimeter [8].

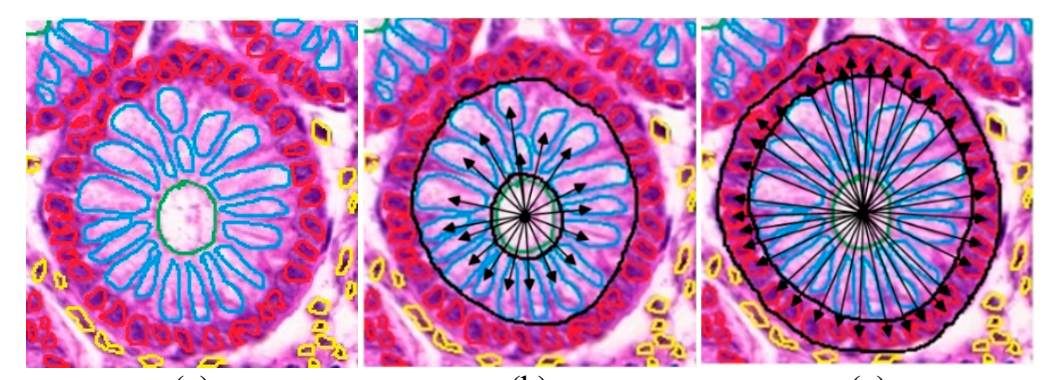

(a) (b) (c)

Figure 7. (a) A single normal colon gland with demarcated lumen, epithelial cells and nuclei; area features are computed from epithelial cells, lumen and nuclei, $(\mathbf{b})$ distance from the centroid of lumen to the centroid of each epithelial cell, (c) distance from the centroid of lumen to the centroid of each cell nucleus. Figure shows one glandular region cropped from an image captured at $20 \times$ magnification.

\subsubsection{Patch-Based Features}

To extract an intermediate level of detail between the image- and gland-based features, the input image was divided into multiple patches of fixed sizes [33,34]. Random subsets of these patches were used to compute the same texture features as those of image-based features.

\subsection{Hierarchical Classification Framework}

Support vector machines, extensively used in the past in clinical studies [35,36], were used to construct various classifiers employed in a hierarchical classification framework used in this study (Figure 2). In the first classification layer, patch-based features from subsets of patches were used to develop multiple weak classifiers based on the radial basis function (RBF) kernel of SVM. The probability estimate of the weak classifier (ProbabilityScore_2) with the highest confidence interval was used in the next step. The other individual features, i.e., image- and gland-based features, were directly classified by using the RBF kernel of SVM and the output probability estimates (ProbabilityScore_1 and ProbabilityScore_3) were used in the next step. In the second classification layer, another ensemble classification model (meta-classification) was built using linear, RBF and sigmoid kernels of SVM as weak classifiers by using probability estimates (ProbabilityScore_1, ProbabilityScore_2, and ProbabilityScore_3) as features. The output of these weak classifiers was combined through majority voting providing the final classification. For all the classification experiments within each dataset, 10-fold cross-validation was used and system parameters were adjusted based on the training data using a grid search mechanism. For quantitative performance evaluation, multiple assessment measures were computed in order to validate the reliability of results.

We have selected the top-most 5 features based on the weight assigned by the classifier, and compared the values of these features in normal and malignant groups. Boxplots of these features based on data from all the patients were created with the group type on $\mathrm{x}$-axis and its value on $\mathrm{y}$-axis (Figure 5). The p-values were then calculated using Wilcoxin rank test [37], and corrected using Bonferroni correction [38]. 


\section{Conclusions}

We present here an ensemble classification framework based on robust image features for accurate colon cancer detection and grading. The proposed methodology was validated both within and between two colon cancer datasets by investigating the performance of individual as well as ensemble classifiers. Our results show that the ensemble classification methodology produces robust classification and reinforces the performance of individual classifiers by a significant margin. The reliability of the proposed methodology between the two datasets suggests broader applicability across diverse clinical settings. With further prospective validation, our method may prove to be a useful tool to quickly, accurately, and reproducibly analyze colon cancer specimens.

Author Contributions: Conceptualization, S.R.; Data curation, S.R.; Formal analysis, S.R., M.A.I., A.C., and T.K.; Methodology, S.R.; Project administration, S.R.; Resources, S.R.; Software, S.R.; Supervision, T.N., M.B.; Validation, S.R., M.A.I., and A.C.; Writing—original draft, S.R.; Writing-review and editing, S.R., M.A.I., A.C., T.K., M.B, and T.N.

Funding: This dataset received no external funding.

Conflicts of Interest: The authors declare no conflict of interests.

\section{References}

1. Cross, S.E.; Jin, Y.S.; Rao, J.; Gimzewski, J.K. Nanomechanical analysis of cells from cancer patients. Nat. Nanotechnol. 2007, 2, 780-783. [CrossRef] [PubMed]

2. Backes, Y.; Moons, L.M.; Novelli, M.R.; van Bergeijk, J.D.; Groen, J.N.; Seerden, T.C.; Schwartz, M.P.; de Vos Tot Nederveen Cappel, W.H.; Spanier, B.W.; Geesing, J.M.; et al. Diagnosis of T1 colorectal cancer in pedunculated polyps in daily clinical practice: A multicenter study. Mod. Pathol. 2017, 30, $104-112$. [CrossRef] [PubMed]

3. Rathore, S.; Iftikhar, M.A.; Hussain, M.; Jalil, A. Classification of colon biopsy images based on novel structural features. In Proceedings of the 2013 IEEE 9th International Conference on Emerging Technologies (ICET), Islamabad, Pakistan, 9-10 December 2013; pp. 1-6.

4. Doyle, S.; Madabhushi, A.; Feldman, M.; Tomaszeweski, J. A boosting cascade for automated detection of prostate cancer from digitized histology. Med. Image Comput. Comput. Assist. Interv. 2006, 9 Pt 2, 504-511.

5. Khan, A.M.; Rajpoot, N.; Treanor, D.; Magee, D. A nonlinear mapping approach to stain normalization in digital histopathology images using image-specific color deconvolution. IEEE Trans. Biomed. Eng. 2014, 61, 1729-1738. [CrossRef] [PubMed]

6. Gurcan, M.N.; Boucheron, L.E.; Can, A.; Madabhushi, A.; Rajpoot, N.M.; Yener, B. Histopathological image analysis: A review. IEEE Rev. Biomed. Eng. 2009, 2, 147-171. [CrossRef]

7. Rathore, S.; Hussain, M.; Ali, A.; Khan, A. A recent survey on colon cancer detection techniques. IEEE/ACM Trans. Comput. Biol. Bioinform. 2013, 10, 545-563. [CrossRef]

8. Rathore, S.; Hussain, M.; Khan, A. Automated colon cancer detection using hybrid of novel geometric features and some traditional features. Comput. Biol. Med. 2015, 65, 279-296. [CrossRef]

9. Altunbay, D.; Cigir, C.; Sokmensuer, C.; Gunduz-Demir, C. Color graphs for automated cancer diagnosis and grading. IEEE Trans. Biomed. Eng. 2010, 57, 665-674. [CrossRef]

10. Ozdemir, E.; Gunduz-Demir, C. A hybrid classification model for digital pathology using structural and statistical pattern recognition. IEEE Trans. Med. Imaging 2013, 32, 474-483. [CrossRef]

11. Rathore, S.; Hussain, M.; Iftikhar, M.A.; Jalil, A. Novel structural descriptors for automated colon cancer detection and grading. Comput. Methods Progr. Biomed. 2015, 121, 92-108. [CrossRef]

12. Chaddad, A.; Daniel, P.; Niazi, T. Radiomics Evaluation of Histological Heterogeneity Using Multiscale Textures Derived From 3D Wavelet Transformation of Multispectral Images. Front. Oncol. 2018, 8, 96. [CrossRef] [PubMed]

13. Haj-Hassan, H.; Chaddad, A.; Harkouss, Y.; Desrosiers, C.; Toews, M.; Tanougast, C. Classifications of Multispectral Colorectal Cancer Tissues Using Convolution Neural Network. J. Pathol. Inform. 2017, 8, 1. [PubMed]

14. Chaddad, A.; Tanougast, C. Texture Analysis of Abnormal Cell Images for Predicting the Continuum of Colorectal Cancer. Anal. Cell Pathol. 2017, 2017, 8428102. [CrossRef] [PubMed] 
15. Ronneberger, O.; Fischer, P.; Brox, T. U-Net: Convolutional Networks for Biomedical Image Segmentation. arXiv 2015, arXiv:1505.04597.

16. Gunduz-Demir, C.; Kandemir, M.; Tosun, A.B.; Sokmensuer, C. Automatic segmentation of colon glands using object-graphs. Med. Image Anal. 2010, 14, 1-12. [CrossRef] [PubMed]

17. Graham, S.; Chen, H.; Gamper, J.; Dou, Q.; Heng, P.-A.; Snead, D.; Tsang, Y.W.; Rajpoot, N. MILD-Net: Minimal Information Loss Dilated Network for Gland Instance Segmentation in Colon Histology Images. Med. Image Anal. 2019, 52, 199-211. [CrossRef]

18. Kainz, P.; Pfeiffer, M.; Urschler, M. Semantic Segmentation of Colon Glands with Deep Convolutional Neural Networks and Total Variation Segmentation. arXiv 2015, arXiv:1511.06919.

19. Fu, H.; Qiu, G.; Shu, J.; Ilyas, M. A novel polar space random field model for the detection of glandular structures. IEEE Trans. Med. Imaging 2014, 33, 764-776. [CrossRef]

20. Li, W.; Manivannan, S.; Akbar, S.; Zhang, J.; Trucco, E.; McKenna, S.J. Gland segmentation in colon histology images using hand-crafted features and convolutional neural networks. In Proceedings of the 2016 IEEE 13th International Symposium on Biomedical Imaging (ISBI), Prague, Czech Republic, 13-16 April 2016.

21. Chen, H.; Qi, X.; Yu, L.; Dou, Q.; Qin, J.; Heng, P.A. DCAN: Deep contour-aware networks for object instance segmentation from histology images. Med. Image Anal. 2017, 36, 135-146. [CrossRef]

22. Masood, K.; Rajpoot, N. Texture based classification of hyperspectral colon biopsy samples using CLBP. In Proceedings of the IEEE International Symposium on Biomedical Imaging: From Nano to Macro, Boston, MA, USA, 28 June-1 July 2009; pp. 1011-1014.

23. Rathore, S.; Hussain, M.; Iftikhar, M.A.; Jalil, A. Ensemble classification of colon biopsy images based on information rich hybrid features. Comput. Biol. Med. 2014, 47, 76-92. [CrossRef]

24. Awan, R.; Sirinukunwattana, K.; Epstein, D.; Jefferyes, S.; Qidwai, U.; Aftab, Z.; Mujeeb, I.; Snead, D.; Rajpoot, N. Glandular Morphometrics for Objective Grading of Colorectal Adenocarcinoma Histology Images. Sci. Rep. 2017, 7, 16852. [CrossRef] [PubMed]

25. Chaddad, A.; Tanougast, C.; Golato, A.; Dandache, A. Carcinoma cell identification via optical microscopy and shape feature analysis. J. Biomed. Sci. Eng. 2013, 6, 1029-1033. [CrossRef]

26. Rathore, S.; Hussain, M.; Khan, A. GECC: Gene Expression Based Ensemble Classification of Colon Samples. IEEE/ACM Trans. Comput. Biol. Bioinform. 2014, 11, 1131-1145. [CrossRef] [PubMed]

27. Rathore, S.; Iftikhar, M.A. CBISC: A Novel Approach for Colon Biopsy Image Segmentation and Classification. Arab. J. Sci. Eng. 2016, 41, 5061-5076. [CrossRef]

28. Tommelein, J.; Verset, L.; Boterberg, T.; Demetter, P.; Bracke, M.; de Wever, O. Cancer-associated fibroblasts connect metastasis-promoting communication in colorectal cancer. Front. Oncol. 2015, 5, 63. [CrossRef]

29. Becht, E.; de Reynies, A.; Giraldo, N.A.; Pilati, C.; Buttard, B.; Lacroix, L.; Selves, J.; Sautes-Fridman, C.; Laurent-Puig, P.; Fridman, W.H. Immune and Stromal Classification of Colorectal Cancer Is Associated with Molecular Subtypes and Relevant for Precision Immunotherapy. Clin. Cancer Res. 2016, 22, 4057-4066. [CrossRef]

30. Sirinukunwattana, K.; Pluim, J.P.; Chen, H.; Qi, X.; Heng, P.A.; Guo, Y.B.; Wang, L.Y.; Matuszewski, B.J.; Bruni, E.; Sanchez, U.; et al. Gland segmentation in colon histology images: The glas challenge contest. Med. Image Anal. 2017, 35, 489-502. [CrossRef]

31. Sirinukunwattana, K.; Snead, D.R.; Rajpoot, N.M. A Stochastic Polygons Model for Glandular Structures in Colon Histology Images. IEEE Trans. Med. Imaging 2015, 34, 2366-2378. [CrossRef]

32. Haralick, R.M.; Shanmugam, K.; Dinstein, I.H. Textural features for image classification. IEEE Trans. Syst. Man Cybern. 1973, 3, 610-621. [CrossRef]

33. Liu, M.; Zhang, D.; Shen, D. Semble sparse classification of Alzheimer disease. Neuroimage 2012, 60, $1106-1116$. [CrossRef]

34. Rathore, S.; Iftikhar, M.A.; Hassan, M. Ensemble Sparse Classification of Colon Cancer. In Proceedings of the 2016 International Conference on Frontiers of Information Technology (FIT), Islamabad, Pakistan, 19-21 December 2016.

35. Fujima, N.; Shimizu, Y.; Yoshida, D.; Kano, S.; Mizumachi, T.; Homma, A.; Yasuda, K.; Onimaru, R.; Sakai, O.; Kudo, K.; et al. Machine-Learning-Based Prediction of Treatment Outcomes Using MR Imaging-Derived Quantitative Tumor Information in Patients with Sinonasal Squamous Cell Carcinomas: A Preliminary Study. Cancers 2019, 11, 800. [CrossRef] [PubMed] 
36. Rehman, O.; Zhuang, H.; Ali, A.M.; Ibrahim, A.; Li, Z. Validation of miRNAs as Breast Cancer Biomarkers with a Machine Learning Approach. Cancers 2019, 11, 431. [CrossRef] [PubMed]

37. Wilcoxon, F. Individual comparisons by ranking methods. Biom. Bull. 1945, 1, 80-83. [CrossRef]

38. Dunn, O.J. Estimation of the Means for Dependent Variables. Ann. Math. Stat. 1958, 29, 1095-1111. [CrossRef]

(C) 2019 by the authors. Licensee MDPI, Basel, Switzerland. This article is an open access article distributed under the terms and conditions of the Creative Commons Attribution (CC BY) license (http://creativecommons.org/licenses/by/4.0/). 